\title{
DISTRIBUTION OF ABO AND RH BLOOD GROUPS IN HIV SEROPOSITIVES AT AN INTEGRATED COUNSELING AND TESTING CENTRE IN KARNATAKA, INDIA
}

\author{
Banu $\mathrm{A}^{1}$, Ahmed SM${ }^{1}$, Shastri $\mathrm{S}^{2}$ \\ ${ }^{1}$ Bowring and Lady Curzon Hospital, Bangalore Medical College and Research Institute, Bangalore Karnataka, India \\ ${ }^{2}$ State AIDS Clinical Expert Panel Member for ART, Technical resource Group, NACO
}

\begin{abstract}
Introduction: $A B O$ blood group system was the first human blood group system to be discovered. Subsequent to the discovery of blood groups by Landsteiner and advancement in its study many workers tried to find out associations between blood groups and the incidence of various diseases. The objective of the study was to create a blood group database which would probably help in transfusion services and find out the distribution of blood groups in the seropositive population.

Methodology: Blood groups were ascertained for 1809 patients who were HIV seropositive enrolled at ICTC in a tertiary care teaching hospital in Karnataka, India from April 2004 to January 2010 using the simple tile method. The results were compiles and statistically analyzed.

Results: Blood grouping was done for 1809 patients, out of which 1749 (96.68\%) were adults and $60(3.32 \%)$ were paediatric patients. O Rhesus positive was the most prevalent blood group in both adult $(40.13 \%)$ and paediatric (43.33\%) seropositives. B Rhesus positive was next commonest group in adults $(26.12 \%)$ and $A$ Rhesus positive in paediatric(30.0\%). AB Rhesus negative (Adults- $0.34 \%$ and Paediatric-0\%) blood group was the least prevalent in the study population.

Conclusion: It is important to create blood group database for the Indian seropositives population, to know any probable association between blood group and HIV infection. This study is an attempt to create a blood group database in a modestly large seropositive which would play a vital role in transfusion services and future research. Larger nation-wide studies would be required to substantiate any association between blood groups and HIV infection.
\end{abstract}

Key words: HIV seropositive, ABO, Rhesus blood groups.

\section{INTRODUCTION}

ABO blood group system was the first human blood group system to be discovered. ${ }^{1}$ Subsequent to the discovery of blood groups by Landsteiner and advancement in its study many workers tried to

\begin{tabular}{l}
\hline Correspondence: \\
Dr. Asima Banu \\
34/1 Sree Ram Mandir Road \\
Basavangudi, Bangalore - 560004 \\
E-mail: asima.banu@gmail.com \\
\hline
\end{tabular}

find out associations between blood groups and the incidence of various diseases. For example, strong associations have been described between $\mathrm{O}$ blood group and peptic ulcer. ${ }^{2} \mathrm{~A}$ blood group and gastric carcinoma. ${ }^{3} A B$ blood group and carcinoma cervix ${ }^{4}$, and so on. In the recent times, according to research done by Swedish scientists, the risk of being infected by Human Immunodeficiency Virus (HIV) may be determined by the presence of the carbohydrate based blood group moiety $P^{k}$. Individuals with high $P^{k}$ levels exhibited a 
greater natural resistance to HIV infection. ${ }^{5 .}$ World literature has few articles describing associations between HIV infection and blood groups .With this background, an attempt was made in this study to create a blood group database in HIV seropositives both adult and paediatric to ascertain the distribution of various $A B O / R h$ blood groups in the study population. The database so created would probably be of help in blood transfusion services particularly in this locality.

\section{METHODOLOGY}

This is a cross sectional study in which blood groups were determined for 1809 HIV seropositives(1749 adults and 60 paediatric) at blood bank of a tertiary care hospital in Karnataka, India, during the period April 2004 to January 2010. Informed consent had been taken from the patients to perform the test. The $A B O$ and Rhesus(Rh) blood grouping was done by a simple tile method. ${ }^{6} \mathrm{~A}$ drop of blood from each subject was placed on a clean white tile in three places. A drop of each of the antisera, anti $A$, and anti $B$ and anti $D$ was added and mixed with each blood sample with the aid of glass rods. Blood groups were determined on the basis of agglutination. $^{7}$

The HIV seropositive status was confirmed by standard ELISA/RAPID tests according to National AIDS Control Organization (NACO) guidelines at the Integrated Counseling and Testing Centre (ICTC). According to the earlier guidelines, HIV testing was confirmed by ELISA. Revised guidelines follow strategy $3 \mathrm{i}$. e. using 3 rapid tests for confirmation of positive status. ${ }^{8}$ Baseline CD4 cell counts were done for all the participants of the study at the Department of Microbiology. The blood group of the control population was obtained from the records of 1168 consecutive personnel maintained at the blood bank of the hospital. Control group was included in this study in order to create a database and also compare the distribution of blood groups among seropositives against the general population so as to determine any significant correlation if present. Age, gender, blood group and CD4 data were compiled and subjected to relevant statistical analysis using SPSS Version 16.

\section{RESULTS}

Blood grouping was done for 1749 adult HIV seropositives, 60 paediatric HIV seropositives between the age group of 18 months and 70 years. The adult seropositive group consisted of $1030(58.9 \%)$ males and $719(41.1 \%)$ females. The paediatric seropositive group consisted of $25(41.7 \%)$ males and $35(58.3 \%)$ females. The control group comprised 1130 adult and 38 paediatric individuals. The adult controls comprised $662(58.6 \%)$ males and $468(41.4 \%)$ females. The paediatric age group consisted of $16(42.1 \%)$ males and $22(57.9 \%)$ females

The adults seropositive group had 1649(94.28\%) Rhesus positive blood group while 100(5.72\%) were Rhesus negative. In the paediatric seropositive group 59(98.53\%) were Rhesus positive and one $(1.66 \%)$ was Rhesus negative. In the adult control group 1067(94.33\%) were Rhesus positive and $64(5.67 \%)$ were Rhesus negative while in the paediatric control group 36(97.36\%) were Rhesus positive while $1(2.63 \%)$ were Rhesus negative as shown in Table1.

\begin{tabular}{|c|c|c|c|c|}
\hline \multirow{2}{*}{$\begin{array}{c}\text { Study } \\
\text { Population }\end{array}$} & \multicolumn{2}{|c|}{ Rhesus Positive } & \multicolumn{2}{|c|}{ Rhesus Negative } \\
\hline & Number & $\%$ & Number & $\%$ \\
\hline $\begin{array}{l}\text { Adult } \\
\text { Seropositive }\end{array}$ & 1649 & 94.28 & 100 & 5.72 \\
\hline $\begin{array}{l}\text { Paediatric } \\
\text { Seropositive }\end{array}$ & 59 & 98.53 & 1 & 1.66 \\
\hline Adult Control & 1066 & 94.33 & 64 & 5.67 \\
\hline $\begin{array}{l}\text { Paediatric } \\
\text { Control }\end{array}$ & 37 & 97.36 & 1 & 2.63 \\
\hline
\end{tabular}

Figure 1 shows that $\mathrm{O}$ Rh positive blood group was the commonest in 702(40.13\%) adult seropositives and $26(43.33 \%)$ in paediatric seropositives as well as $407(34.84 \%)$ in the control group. In the adult seropositives population B Rh positive blood group was the next commonest in $457(26.12 \%)$ followed by A Rh positive blood group in 395(22.58\%). The control group also showed a similar trend with $341(29.19 \%)$ individuals having B Rh positive blood group and $279(23.88 \%)$ having A Rh positive blood group. The paediatric seropositive population 
showed a variation with more number of females than males and $\mathrm{A}$ Rh positive blood group being the next commonest in $17(28.33 \%)$ followed by B Rh positive blood group in 14(23.33\%). Figure 2, 3 and 4 show the gender-wise distribution of blood groups in adult, paediatric seropositive and the control population respectively. In all the three groups $A B$ Rh negative was the least commonly observed blood group.

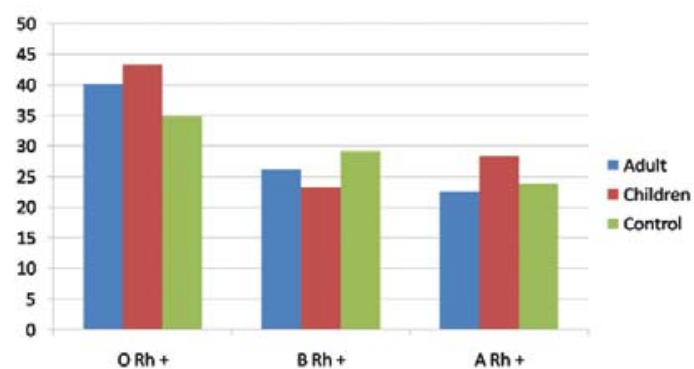

Figure 1. Bar graph showing the percentage ( $Y$ axis) of adult, paediatric and control subjects positive for $\mathrm{O} \mathrm{Rh}+$, $\mathrm{B} R \mathrm{Rh}+$ and $\mathrm{ARh}+$ blood groups

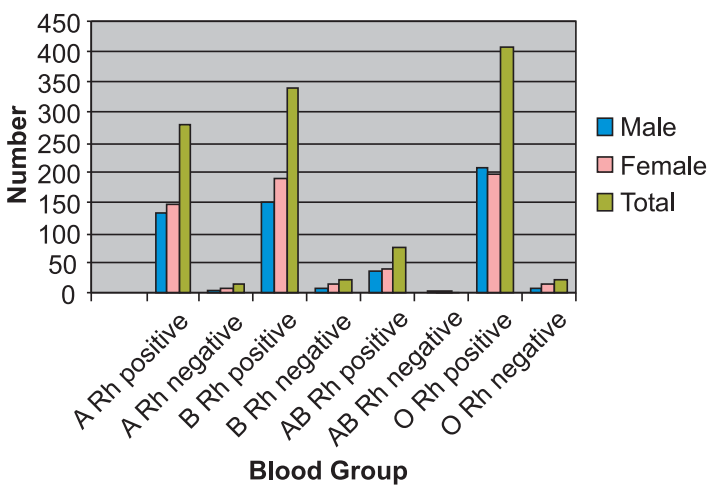

Figure 2. Gender-wise Distribution of Blood Groups in Adult Seropositives

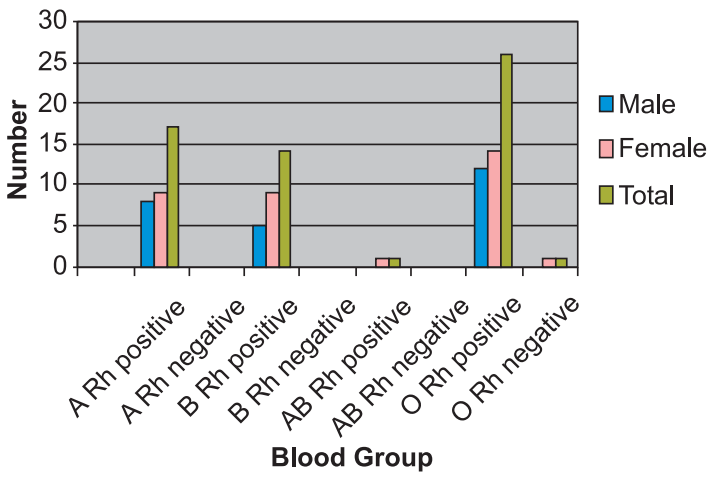

Figure 3. Gender-wise Distribution of Blood Groups in Paediatric Seropositives

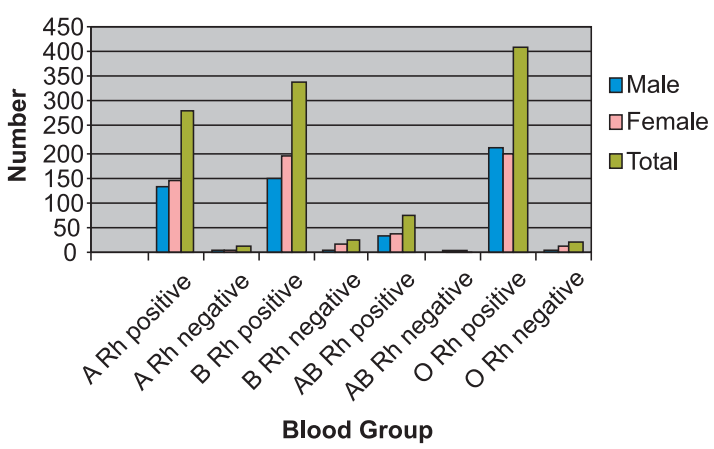

Figure 4. Blood Group Distribution in Control Population

The mean baseline CD4 cell count of the study population was 121.77 cells/ $\mu$ l.

\section{DISCUSSION}

Few studies from the world have researched probable association between blood group and HIV infection. Very few large scale studies have been conducted in India to know the distribution of blood groups of HIV seropositive population. In this study it was observed that O Rh positive was the commonest among the adult $702(40.13 \%)$ and paediatric $26(43.33 \%)$ seropositive population as well as the control $407(34.84 \%)$ population. An Indian study which determined the distribution of blood groups in 104 HIV seropositives observed 0 blood group in $40(38.5 \%)$ individuals which was most common, $A$ blood group was seen in $28(26.9 \%), 26(25 \%)$ had $B$ blood group and 10(9.6\%) had $A B$ blood group. ${ }^{9}$ The current study showed $457(26.12 \%)$ with B Rh positive blood group in adult and $17(28.33 \%$ ) with A Rh positive blood group in paediatric study population, which were the next most prevalent blood groups. In addition, there were more female seropositives than males in the paediatric study group. In a study from Nigeria, HIV status and blood groups determination (Rhesus and $A B O$ groups) in 3691 pregnant women attending antenatal clinic and 1199 non-pregnant women visiting the same institution between 1999 to 2002 were studied. Overall, the prevalence of blood group $\mathrm{O}$ Rh positive was higher than in the general population with highest rate of $62.9 \%$ in HIV positive pregnant women followed by $58.4 \%$ in HIV negative pregnant women and $58.0 \%$ in 
non-pregnant women. No significant difference was observed in blood groups for the three categories of subjects studied showing that no particular blood group type could be linked to the occurrence of HIV infection. ${ }^{10}$ In another Nigerian study which determined $A B O$ blood groups for 216 seropositives, $42.1 \%$ were $O$ group, $11.1 \% A$ group, $9.4 \% B$ group and $8.7 \%$ were $A B$ group and 5 out of $7(71.4 \%)$ infected subjects with HIV-2 only, belonged to blood group AB. ${ }^{11}$ The present study also had one HIV-2 infected individual who was found to be B Rh positive.

ABO histo-blood group antigens have been postulated to modify pathogen spread through the action of natural antibodies and complement. Incorporation of $A B O$ antigens by HIV-1 may affect transmission of virus between individuals of discordant blood groups by interaction with host natural antibody and complement. While much research has been devoted to searching for genetic factors that confer resistance to HIV-1 transmission, the most notable being the MHC and CCR5 loci, little is known about the role that some of the most well-studied blood group polymorphisms may play in viral transmission. ${ }^{12}$ Swedish researchers investigated cell surfaceexpressed $P^{k}$ in HIV infection. They concluded that $P^{k}$ expression strongly influences susceptibility to HIV-1 infection, which implicates $P^{k}$ as a new endogenous cell-surface factor that may provide protection against HIV-1 infection. Individuals with high $P^{k}$ levels exhibited a greater natural resistance to HIV infection. ${ }^{5}$

It is important to create blood group database for the Indian seropositives population, to know about blood group distributions and any probable association between blood group and HIV infection. This study is an attempt to create a blood group database in a modestly large seropositive population which would play a vital role in blood transfusion services and future research protocols. Larger nation-wide studies would be required to substantiate any association between blood groups and HIV infection.

\section{REFERENCES}

1. Mollison PL. Blood transfusion in Clinical Medicine 6th Edition London Blackwell Publications, 1979.

2. Clark CA, Howel-Evans AW, Mc Connell RB, Sheppard C. Secretion of blood group antigens and Peptic ulcer. BMJ 1959;225.

3. Airid I, Bentall H, Roberts JA. Relationship between cancer stomach and the $A B O$ blood groups. BMJ 1953;1:799.

4. Tyagi SP, Tyagi GK, Pradhan AC. ABO blood in relation to carcinoma cervix. Ind $\mathrm{J}$ Med Sci 1967;21:611.

5. Lund $N$, Olsson ML, Ramkumar $S$, Sakac D, Yahalom V, Levene C, Hellberg A, Ma XZ, Binnington $B$, Jung $D$, Lingwood CA, Branch DR. The human $P(k)$ histo-blood group antigen provides protection against HIV-1 infection. Blood. 2009 14;113(20):4980-91.

6. Dacie JV, Lewis SM. Practical Textbook of Haematology 7th Edition Edinburgh. Church Livingstone, 1991.

7. Egesie UG, Egesie OJ, Usar I, johnbull TO. Distribution of $\mathrm{ABO}$, Rhesus blood groups and haemoglobin electrophoresis among the undergraduate students of Niger Delta University Nigeria. Nigerian Journal of Physiological Sciences. 2008; 23(1-2): 5-8

8. NACO ARV Treatment Guidelines Available from: http:// www.nacoonline.org/upload/Publication/ Treatment Care and support/Antiretroviral Therapy Guidelines for HIV-Infected Adults and Adolescents Including Post-exposure.pdf, accessed on November 12, 2009.

9. Sayal SK, Das AL, Nema SK. Study of blood groups in HIV seropositive patients. Indian J Dermatol Venereol Leprol 1996;62:295-7.

10. Nneli RO, Ekpo BO, Ohaeri OC, Egene J. Prevalence of $R h$ and $A B O$ blood groups in HIV seropositive pregnant women in Enugu, Nigeria. Nigerian Journal of Physiological Sciences 2004;19(1-2): 7-9.

11. AbdulazeezAA, Alo EB, Rebecca SN. Carriage rate of Human Immunodeficiency Virus (HIV) infection among different $A B O$ and Rhesus blood groups in Adamawa state, Nigeria. Biomedical Research 2008; 19 (1): 41-44.

12. Neil SJD, McKnight A, Gustafsson K, Weiss RA. HIV-1 incorporates $A B O$ histo-blood group antigens that sensitize virions to complement-mediated inactivation. Blood 2005;105:4693-4699. 